\title{
Dietary fibre intake and constipation in the UK Women's Cohort Study
}

\author{
L. Alrefaai, J. E. Cade and V. J. Burley \\ School of Food Science \& Nutrition, University of Leeds, Leeds LS2 9JT, UK
}

\begin{abstract}
Although it has long been recognised that dietary fibre has an impact on faecal volume, reports of relationships between dietary fibre and incidence of constipation are sparse and inconsistent ${ }^{(1,2)}$. The UK Women Cohort's Study ${ }^{(3)}$ is a population-based prospective cohort study of middle-aged women that we have used here to explore the relationship between dietary fibre intake and risk of constipation.

From 35,372 women who completed a 217-item food frequency questionnaire (FFQ) between 1996 and 1999, approximately 45\% also completed a follow-up questionnaire after an average of 4 years. At follow-up, the women reported frequency of bowel movements and consistency of stools which were used to define constipation. Two sets of criteria were applied; having $\leq 3$ bowel movements (BM) per week (1st definition) and having hard stools and $\leq 3 \mathrm{BM}$ per week (2nd definition). Estimates of dietary fibre intake (non-starch polysaccharides) and main sources of fibre were obtained from the baseline FFQ.

After exclusion criteria were applied (pregnancy, unfeasible energy or fibre intakes, prevalent bowel disease), 10,517 eligible subjects remained for the analysis. Logistic regression was used to explore the relationship between dietary fibre intake at baseline and having a subsequent definition of constipation (using either definition) at follow-up. Two models were used, an unadjusted and a multivariableadjusted model.

Using the first definition of constipation ( $\leq 3 \mathrm{BM} / \mathrm{wk}), 589(5.9 \%)$ of the women were constipated whereas just $192(1.8 \%)$ women met the criteria for the second definition $(\leq 3 \mathrm{BM} / \mathrm{wk} \&$ hard stool). The odds of being constipated at follow-up were significantly lower (by $40-50 \%)$ in women in the highest fibre intake quintile compared with the lowest consumers using the first and second constipation definitions respectively (see table). All sources of fibre were inversely associated with lower odds of constipation using the first definition, however, only vegetable fibre was inversely associated with constipation using the second definition.
\end{abstract}

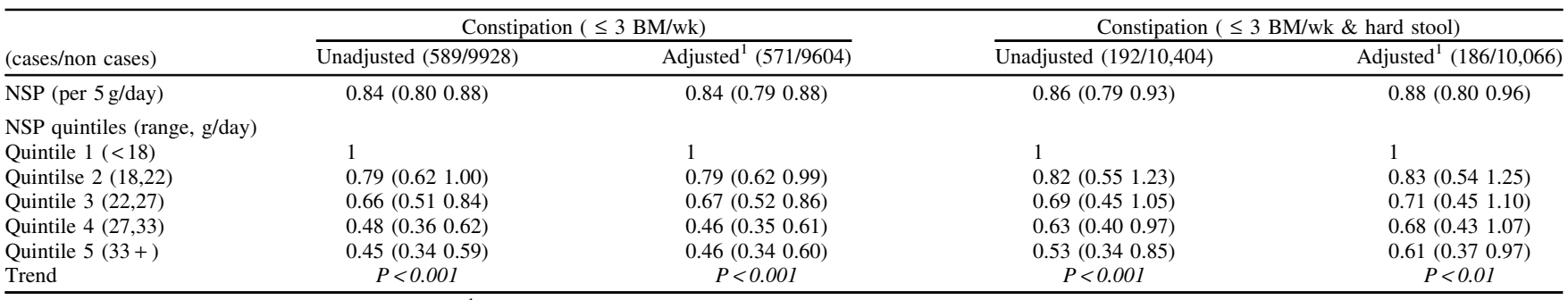

*Values are odds ratios (95\% confidence intervals) ${ }^{1}$ adjusted for smoking, alcohol, age, body mass index and exercise habits.

In this cohort of health conscious women, with each $5 \mathrm{~g}$ per day increment of dietary fibre consumed, the odds of being constipated were reduced by 12 to $16 \%$. In order to maintain good bowel health, these data lend support to public health recommendations that encourage consumption of high fibre food sources.

1. Dukas L, Willett WC, Giovannucci EL (2003) Am J Gastroenterol 98, 1790-6.

2. Markland AD, Palsson O, Goode PS et al. (2013) Am J Gastroenterol (2013) April 9.

3. Cade JE, Burley VJ, Greenwood DC et al. (2004) Public Health Nutr (2004) 7, 871-8. 